\title{
Systemic Thrombolyis within 24 Hours of Major Cardiac Surgery; When the Risk to Life outweighs the Haemorrhage
}

\author{
Mahmoud M Abdelaziz*, Nasar Ahmad and John S Billing \\ Department of Cardiothoracic Surgery, Heart and Lung Centre, New Cross Hospital, Wolverhampton, UK
}

*Corresponding author: Mahmoud M Abdelaziz, Department of Cardiothoracic Surgery, Heart and Lung Centre, New Cross Hospital, Wolverhampton, UK WV10 0QP, UK.
Received Date: August 31, 2018

Published Date: October 04, 2018

\section{Introduction}

Ischaemic stroke after cardiac surgery is well recognised devastating complication. The incidence of stroke usually varies based on patients' risk factors and peri-operative related factors and can be as high as 6\% [1]. Due to the high risk of Intracerebral and systemic haemorrhage, systemic thrombolysis is contraindicated within 14 days after any major surgery including open heart surgery [2]. Given the high risk of bleeding, unsurprisingly there has not been any research articles into the bleeding rate of systemic thrombolysis after major surgery.

We present a case of using systemic thrombolysis within 24 hours post major open-heart surgery for treating acute ischaemic stroke.

\section{Clinical Summary}

An 81-year-old man presented with increasing shortness of breath and previous hospital admission with congestive heart failure and pulmonary oedema. Echocardiography confirmed severe mitral and tricuspid valve regurgitation, moderately elevated pulmonary artery pressures (PASP $56 \mathrm{mmHg}$ ) and preserved left ventricular function. His risk factors of heart disease included primary hypertension and hypercholesterolaemia. He underwent median sternotomy, cardiopulmonary bypass with systemic cooling to $30^{\circ} \mathrm{C}$, Mitral valve and Tricuspid valve repair. He was transferred to the intensive care unit as per routine and immediate postoperative recovery was unremarkable. 13 hours after the operation the patient suddenly became unresponsive with poor respiratory effort and Glasgow coma score (GCS) deteriorated from normal to 3 for which he required emergency re-intubation and ventilation. His Pupils were unequal.

Computer Tomography angiography (CTA) scan of the brain did not show any obvious areas of infarcts or bleeding, but clinical assessment by expert neurology physician concluded the likelihood of major brain stem stroke which carried very high risk to life and poor prognosis if left untreated. Targeted cerebral intra-arterial thrombolysis was not feasible based on CT findings and the anatomy of the affected area. Upon discussion with the surgical team, cardiac anaesthetic team and patient's family, the decision was made to treat the patient with systemic thrombolysis given the likelihood of not surviving this insult if left untreated and accept the risk of major bleeding event.

The patient was treated with $0.9 \mathrm{mg} / \mathrm{kg}$ of Alteplase with $10 \%$ of the dose as bolus and the remaining dose as an intravenous infusion over 60 minutes. There was no increase of drainage through the pleural or mediastinal drains or chest x-ray evidence of pleural effusion. Repeat CT brain 24 hours after thrombolysis did not reveal any abnormalities/intracerebral haemorrhage. The patient haemoglobin remained stable and there was no haematological evidence of blood loss after thrombolysis. Within the next 24-48 hours he shows good neurological recovery and was able to communicate and walk with assistance and walking aids 15 days after his operation. He was discharged 42 days postoperatively for further neurological rehabilitation in specialised centre. The patient was reviewed in the outpatient follow up clinic and was able to mobilise and walk independently with the aid of walking stick only.

\section{Discussion}

Major haemorrhage is the main complication after systemic thrombolysis. To our knowledge, there are no reports of systemic thrombolysis within 2 weeks of major cardiac surgery presumably due to high risk of bleeding. Intra-arterial thrombolysis (ITA) has been used after surgery but haemorrhage remained a major risk. In Their review, Chalela et al. reported the risk of bleeding with (due 
IAT after surgery to be as high as $25 \%$ with $8 \%$ death rates in their cohort [3].

In the case presented, intra-arterial thrombolysis was not a possible option due to anatomical reasons and lack of clear area of arterial infarct on the radiological imaging. Given the nature of the patient's presentation and complete loss of vital central functions, it was likely that the outcome is unfavourable. With multidisciplinary team approach and discussion with neurological physicians and patient's family; decision was to proceed with systemic thrombolysis and accept the risk of bleeding.

\section{Conclusion}

To our knowledge this is the only case in the literature whereby systemic thrombolysis was administered after major cardiac surgery. Despite the risk of bleeding in this case, the benefits outweigh the risks for thrombolysis. The use of thrombolysis after cardiac surgery can be considered as a salvage treatment in selected cases.

\section{References}

1. Roach GW, Kanchuger M, Mangano CM, Newman M, Nussmeier N, et al. (1996) Adverse cerebral outcomes after coronary bypass surgery. Multicenter study of perioperative ischemia research group and the ischemia research and education foundation investigators. $\mathrm{N}$ Engl J Med 335(25): 1857-1863.

2. Hacke W, Kaste M, Bluhmki E, Brozman M, Davalos A, et al. (2008) Thrombolysis with alteplase 3 to 4.5 hours after acute ischemic stroke. N Engl J Med 359(13): 1317-1329.

3. Chalela JA, Katzan I, Liebeskind DS, Rasmussen P, Zaidat O, et al. (2001) Safety of intra-arterial thrombolysis in the postoperative period. Stroke 32(6): 1365-1369. 